\title{
Preventive behavior of Opisthorchiasis among people in Ubon Ratchathani province, Thailand
}

\author{
Kaewjai Maleelai ${ }^{1 *}$, Sasimaphon Chaikaew², Aungsumalin Nakham ${ }^{3}$, Jinda Khumkaew ${ }^{1}$
}

\begin{abstract}
Background: Although we are in the era of the 21st-century, some people's lifestyles are still unchanged. People are still eating crude or undercooked fish, which puts them at risk for opisthorchiasis infection. This study aimed to measure knowledge and prevention behavior regarding opisthorchiasis among people resident in Ubon Ratchathani, the northeastern region of Thailand.
\end{abstract}

Methods: A cross-sectional study was conducted from 2nd October 2018 to 26 th December 2018. The data was collected via a self-administered household survey using a systematic random sampling technique. Univariate and bivariate analyses such as the chi-square test were performed. SPSS version 16 was used to analyze the data, and the significance of less than 0.05 was considered.

Results: Four hundred and fifteen households have completed the questionnaire. Most of the participants were female $(50.1 \%)$, aged $15-59$ years old $(75.3 \%)$, married $(64.8 \%)$, had completed elementary school $(46.3 \%)$, worked in agriculture $(31.6 \%)$, and had a monthly salary of on average of 6,525 baht. Most of the respondents reported excellent behavior $(94.2 \%)$ and good knowledge $(80.5 \%)$ to prevent opisthorchiasis, respectively. However, there was no significant association between participants' characteristics and the related behavior to prevent opisthorchiasis $(p>0.05)$.

Conclusion: Despite efforts to educate the community, some people lack preventive knowledge and exhibit behavior associated with a higher risk of opisthorchiasis.

Keywords: Preventive Behavior, knowledge, Opisthorchiasis, Cholangiocarcinoma, Thailand

\section{Background}

Opisthorchiasis is a parasitic infection caused by a foodborne (liver fluke) trematode Opisthorchis viverrini [1]. Opisthorchiasis is an endemic disease and a major public health issue in Southeast Asia, particularly Thailand, the Lao People's Democratic Republic (LAO PDR), Vietnam, and Cambodia [25]. In Southeast Asia, an estimated 10 million people are infected with Opisthorchis viverrini, and approximately 680 million people are at risk of infection [6]. Humans are infected with the Opisthorchis species of liver fluke parasites when they eat parasite-infected raw or undercooked fish, crabs, or crayfish. The liver, gallbladder, and bile duct are the target organs. Most infected people do not show any symptoms; however, severe symptoms and sickness appeared among the untreated infections. A long-term Opisthorchiasis infection can last up to 25-30 years, depending on the parasite's lifetime [7].

*Correspondence: kaewjai@scphub.ac.th

${ }^{1}$ Sirindhorn College of Public Health, Ubon Ratchathani, Faculty of Public Health and Allied Health Sciences, Praboromarajchanok Institute, Thailand.

A full list of author information is available at the end of the article.
Liver fluke infections develop persistent biliary inflammation or chronic inflammation leading to oxidative DNA damage of the infected biliary epithelium, peridural fibrosis, and malignant transformation of liver cells [8]. Opisthorchis-associated cholangiocarcinoma is a fatal cancer of the bile duct $\square 9$ ]. Data on opisthorchiasis and cholangiocarcinoma from Cambodia, Laos, and Vietnam are rare, compared to Thailand, which has a high rate of opisthorchiasis and the world's highest rate of cholangiocarcinoma [10-12]. Sripa et al. [13] reported that around 8 million individuals are infected with liver fluke. Xia et al. [14] indicated that the northeastern region of Thailand has the highest rates of cholangiocarcinoma, with an approximate rate of 85 cases per 100,000 people each year. Several previous studies [15-23] agreed that the O. viverrini infested area in northeastern Thailand includes Khon Kaen province, Yasothon province, Nakhon Ratchasima province, Nakhon Phanom, Sakhon Nakhon, Nong Khai, Udon Thani province, and Ubon Ratchathani province. Thaewnongiew et al. [24] and Khuntikeo et al. [25] reported that people in northeastern Thailand have an exceptionally high incidence of opisthorchiasis infection due to the long-time consumption of traditional raw food prepared 
from cyprinid fish (uncooked, fermented, or undercooked) [24, 25]. The authors also had emphasized that changing the raw seafood consumption behavior and educating people to have accurate knowledge and more awareness about risk factors for cholangiocarcinoma are important ways to prevent the disease. This study aimed to explore the level of prevention behavior for opisthorchiasis among people in northeastern Thailand and the related factors.

\section{Methods}

\section{Study population}

A cross-sectional study was conducted from 2nd October 2018 to 26th December 2018. A self-administered and semistructured questionnaire was recruited to collect the data from people living in the That subdistrict, Warin Chamrap District in Ubon Ratchathani Province, Thailand. A letter was written by the research group to the Bua Wat Subdistrict Health Promoting Hospital requesting permission to collect information using systematic random sampling from an eligible 1,439 households.

\section{Inclusion and exclusion criteria}

All Thai people aged 15 years and above, living in That subdistrict, Warin Chamrap district, Ubon Ratchathani province, Thailand, able to read and write and willing to participate have been included in the study. The illiterate, non-Thai, from outside the region, aged lower than 15 years old, and those unwilling to participate are excluded from the study.

\section{Sample size}

The sample size was computed using Raosoft software. (http://www.raosoft.com/samplesize.html?nosurvey) based on the previous study [26], the prevalence of opisthorchiasis of $52.0 \%$, and marginal error of $5.0 \%$, and a confidence interval of $95 \%$. With non-response correction $=10 \%$, the total sample size was 415 households in this study.

Systematic random sampling was recruited to collect the data. We divided the total number of households by 11 villages within the sample group proportional to the household size of each village. The sample groups in each are as follows:

$\left.\mathrm{n}_{\mathrm{n}}=\left(\mathrm{n} \times \mathrm{n}_{\mathrm{h}}\right) / \mathrm{N}\right)$

$\mathrm{n}_{\mathrm{n}}=$ Random number of citizens in each village

$\mathrm{n}_{\mathrm{h}}=$ The number of people in each village

$\mathrm{n}=$ The total number of sample sizes calculated according to the sample size calculation formula.

$\mathrm{N}=$ Total population.

\section{Questionnaire}

The study tool included three parts:

Part 1: Characteristics of a sample such as age, gender, marital status, level of education, occupation, and monthly income.

Part 2: Preventive behavior of opisthorchiasis. There were three levels of the questionnaire in the rating scale. Respondents could only choose one answer. The point was assigned based on the response and type of item (positive versus negative). To categorize raw fish consumption behavior, we used the following criteria: "Regularly" means consuming raw fish more than three times a month, "Sometimes" means consuming raw fish 1-2 times a month, and "Never" means not eating at all. The scoring criteria for items $1-10$ of the opisthorchiasis preventive behavior questionnaire were: positive item (regularly prevention behavior mark three points, sometimes preventive behavior mark 2 points, never preventive behavior mark 1 point), While negative item (regularly prevention behavior mark 1 point, sometimes preventive behavior mark 2 points, never preventive behavior mark 3 points). Items $7,8,9$, and 10 were positive, while items $1,2,3,4,5$, and 6 were negative. The total score of the ten items ranges from 10-30, which has been subdivided into three levels: "good", "moderate", and "low". To determine the cutoffs for the three-level, we divided the range (Highest score - lowest score) by 3. Thus, the three levels were the score in the range of 21-30, "good behavior", the score is in the range of 10-20, "bad behavior" and, the score is in the range of less than 10, "low behavior". Part 3: Knowledge on prevention of opisthorchiasis. We have defined the scoring criteria in the knowledge test of opisthorchiasis as follows: "correct answer" mark 1 point and "incorrect answer" mark 0 points. Item 1, 2, 3, 4, 5, 6, 8, 9 were positive, while item 7 was negative. The total score of the nine items, ranging from 0-9 points. Interpretation of the total scores divided into three levels: "good", "moderate" and, "low" as follows: the score ranges from 7-9, good level, the score ranges from 4-6, moderate level and the score ranges from 0-3, low level. Before the study, the questionnaire was test piloted among 30 people (not included in the study). All questions were in local language. The Cronbach's alpha coefficient was 0.7 .

\section{Statistical analysis}

Data collected were analyzed using Statistical Package for Social Science (SPSS) program version 20.0 (SPSS Inc., Chicago IL, USA). We analyzed data using descriptive statistics, including mean, standard deviations, number, and percentages. Significant factors predicting the level of preventive behavior for opisthorchiasis ( $p$-value $<0.05$ ) were tested using the Chi-square test. An alpha level of $p<0.05$ is considered to be statistically significant.

\section{Results}

\section{Sociodemographic factors}

The mean age of respondents was 45.0 (SD 17.4) and in the range (15-71). Most of the respondents $(50.2 \%)$ were females, married $(64.8 \%)$, primary school $(46.3 \%)$, working in agricultural field (31.6\%), and had monthly income (51.3\%) of more than 5,000 baths (local currency in Thai bath and 33.02 Thai baths it is equivalent in 1 US dollars, date of exchange on 17 October 2018).

\section{Preventive behavior for Opisthorchiasis}

Most participants reported preventive behavior for opisthorchiasis at a "good" level (391, 94.2\%). However, a small proportion reported preventive behavior for opisthorchiasis at 'not good" levels $(24,5.8 \%)$. The highest percentage of respondents $(93.7 \%)$ reported that they regularly use a hygienic toilet, wash their hands after defecation (74.7\%), before cooking $(87.0 \%$ ), and before dining $(86.7 \%)$. Most of the participants reported "sometimes" eating raw fermented Jaew Bong (66.0\%), raw Som Pra, Som Pra Noi (53.5\%), and raw Pra Jom (53.0\%). Varying proportions of participants reported "never" eating raw Mum Pra (63.1\%), raw fish (Lab pra/ Koi pra) (52.5\%), and raw Pra Jom (44.1\%) (Table 2). 
Table 1: Frequency distribution of categorized sociodemographic variables of respondents $(n=415)$

\begin{tabular}{|c|c|c|c|c|}
\hline Participant characteristics & Categories & $\mathrm{N}(\%)$ & Good N(\%) & Not Good N(\%) \\
\hline \multirow[t]{2}{*}{ Gender } & Male & $207(49.8)$ & $192(46.2)$ & $15(3.6)$ \\
\hline & Female & $208(50.2)$ & $199(48.0)$ & $9(2.2)$ \\
\hline \multirow[t]{4}{*}{ Age } & $15-29$ & $104(25.1)$ & $95(22.9)$ & $9(2.2)$ \\
\hline & $30-44$ & $104(25.0)$ & $98(23.6)$ & $6(1.4)$ \\
\hline & $45-59$ & $104(25.0)$ & $98(23.6)$ & $6(1.4)$ \\
\hline & $>60$ & $103(24.8)$ & $100(24.1)$ & $3(0.7)$ \\
\hline \multirow[t]{4}{*}{ Marital Status } & Single & $104(25.1)$ & $100(24.1)$ & $4(1.0)$ \\
\hline & Married & $269(64.8)$ & $249(60.0)$ & $20(4.8)$ \\
\hline & Divorced & $10(2.4)$ & $10(2.4)$ & $0(0.0)$ \\
\hline & Widowed & $32(7.7)$ & $32(7.7)$ & $0(0.0)$ \\
\hline \multirow[t]{7}{*}{ Educational attainment } & Less than primary school & $10(2.4)$ & $8(1.9)$ & $2(0.5)$ \\
\hline & Primary school & $192(46.3)$ & $181(43.6)$ & $11(2.7)$ \\
\hline & Junior High School & $89(21.4)$ & $83(20.0)$ & $6(1.4)$ \\
\hline & High school diploma & $80(19.3)$ & $76(18.3)$ & $4(1.0)$ \\
\hline & Diploma/Vocational certificate & $17(4.1)$ & $17(4.1)$ & $0(0.0)$ \\
\hline & Bachelor's degree & $24(5.8)$ & $23(5.5)$ & $1(0.2)$ \\
\hline & Post-graduate degree & $3(0.7)$ & $3(0.7)$ & $0(0.0)$ \\
\hline \multirow[t]{8}{*}{ Occupation } & Farmer & $131(31.6)$ & $121(29.2)$ & $10(2.4)$ \\
\hline & Contractors & $88(21.2)$ & $82(19.7)$ & $6(1.4)$ \\
\hline & Trade & $71(17.1)$ & $68(16.4)$ & $3(0.7)$ \\
\hline & Employees & $15(3.6)$ & $15(3.6)$ & $0(0.0)$ \\
\hline & Government officials & $19(4.6)$ & $17(4.1)$ & $2(0.5)$ \\
\hline & Housewife & $50(12.0)$ & $47(11.3)$ & $3(0.7)$ \\
\hline & Student & $29(7.0)$ & $29(7.0)$ & $0(0.0)$ \\
\hline & Others & $12(2.9)$ & $12(2.9)$ & $0(0.0)$ \\
\hline \multirow[t]{4}{*}{ The income per month (Thai baht) } & $<1,000$ & $70(16.9)$ & $68(16.4)$ & $2(0.5)$ \\
\hline & $1,001-3,000$ & $64(15.4)$ & $61(14.7)$ & $3(0.7)$ \\
\hline & $3,001-5,000$ & $68(16.4)$ & $64(15.4)$ & $4(1.0)$ \\
\hline & $>5,000$ & $213(51.3)$ & $198(47.7)$ & $15(3.6)$ \\
\hline
\end{tabular}

Table 2: Preventive behavior for opisthorchiasis $(n=415)$

\begin{tabular}{|l|l|l|l|}
\hline Preventive behavior for opisthorchiasis & Regularly N(\%) & $\begin{array}{l}\text { Sometimes } \\
\mathrm{N}(\%)\end{array}$ & $\begin{array}{l}\text { Never } \\
\mathrm{N}(\%)\end{array}$ \\
\hline 1. You eat raw fish (Lab pra/ Koi pra) & $8(1.9)$ & $189(45.5)$ & $218(52.5)$ \\
\hline $\begin{array}{l}\text { 2. You eat Somtum with raw fermented fish (Pra Ra) / Raw fermented } \\
\text { fish (Pra Ra) }\end{array}$ & $162(39.0)$ & $204(49.2)$ & $49(11.8)$ \\
\hline 3. You eat raw Mum Pra & $11(2.7)$ & $142(34.2)$ & $262(63.1)$ \\
\hline 4. You eat raw Pra Jom & $12(2.9)$ & $220(53.0)$ & $183(44.1)$ \\
\hline 5. You eat raw fermented Jaew Bong & $49(11.8)$ & $274(66.0)$ & $92(22.2)$ \\
\hline 6. You eat raw Som Pra / eat raw Som Pra Noi & $16(3.9)$ & $222(53.5)$ & $177(42.7)$ \\
\hline 7. Before dining, they cleansed their hands & $360(86.7)$ & $52(12.5)$ & $3(0.7)$ \\
\hline 8. Washed their hands before cooking & $361(87.0)$ & $51(12.3)$ & $3(0.7)$ \\
\hline 9. Defecated in a hygienic toilet & $389(93.7)$ & $24(5.8)$ & $2(0.5)$ \\
\hline 10. Washed their hands with soap after defecation & $310(74.7)$ & $98(23.6)$ & $7(1.7)$ \\
\hline
\end{tabular}

Knowledge on preventive of opisthorchiasis

Table 3 shows the results of the knowledge about preventive of opisthorchiasis. Before eating and dining, most of the respondents carefully cleaned the fresh vegetables $(97.1 \%)$ and their hands $(96.9 \%)$ to prevent helminth eggs from entering the body, respectively. A high percentage of participants knew that cooking freshwater fish with scales such as carp and whitefish can prevent opisthorchiasis (95.2\%). The most incorrect answer $(41.2 \%)$ by participants was that about the best way to prevent
Liver fluke disease is to take a helminthic medicine every time after eating raw fish $(41.2 \%)$. The other incorrect answers included that eliminating freshwater mollusks reduces the spread of helminth eggs $(30.6 \%)$, and if a dog continues to defecate, this will cause the distribution of helminth eggs to freshwater mollusks (20.7\%). Most participants knew to prevent opisthorchiasis at a "good" level (334, 80.5\%). Small proportions knew preventive opisthorchiasis at a "moderate" level $(78,18.8 \%)$ and a low level $(3,0.7 \%)$. 
Table 3: The knowledge about prevention of opisthorchiasis $(n=415)$

\begin{tabular}{|l|l|l|}
\hline Knowledge about prevention of opisthorchiasis & $\begin{array}{l}\text { Yes } \\
\mathbf{N}(\%)\end{array}$ & $\begin{array}{l}\text { No } \\
\mathbf{N}(\%)\end{array}$ \\
\hline 1. Consumption of cooked freshwater fish with scales such as carp and whitefish can prevent opisthorchiasis & $395(95.2)$ & $20(4.8)$ \\
\hline 2. Before dining, cleansing their hands helps to prevention helminth eggs from entering the body & $402(96.9)$ & $13(3.1)$ \\
\hline 3. Defecation in the toilets every time as prevents spread of helminth eggs into water resources & $393(94.7)$ & $22(5.3)$ \\
\hline 4. If the dog continues to defecate, this will cause the distribution of helminth eggs to freshwater mollusks & $329(79.3)$ & $86(20.7)$ \\
\hline 5. Eliminating freshwater mollusks reduces the spread of helminth eggs & $288(69.4)$ & $127(30.6)$ \\
\hline 6. Annual stool examinations can be prevention opisthorchiasis & $372(89.6)$ & $43(10.4)$ \\
\hline 7. The best way to prevent opisthorchiasis is to take a medicine every time after eating raw fish & $244(58.8)$ & $171(41.2)$ \\
\hline 8. The most effective way to prevent opisthorchiasis is to stop eating undercooked freshwater fish with scales & $355(85.5)$ & $60(14.5)$ \\
\hline 9. Before eating fresh vegetables, they carefully clean to prevent helminth eggs from entering the body & $403(97.1)$ & $12(2.9)$ \\
\hline
\end{tabular}

The relationship between participant characteristics and level of preventive behavior for opisthorchiasis

Table 4 shows the relationship between participant characteristics and level of preventive behavior for opisthorchiasis. There was no statistically significant association between the personal characteristics of participants and the level of preventive behavior for opisthorchiasis.

Table 4: The relationship between participant characteristics and level of preventive behavior for opisthorchiasis

\begin{tabular}{|c|c|c|c|c|}
\hline Participant characteristics & Categories & $\begin{array}{l}\text { Good } \\
\mathrm{N}(\%)\end{array}$ & $\begin{array}{l}\text { Not Good } \\
\mathrm{N}(\%)\end{array}$ & p-value \\
\hline \multirow[t]{2}{*}{ Gender } & Male & $192(46.2)$ & $15(3.6)$ & 0.203 \\
\hline & Female & $199(48.0)$ & $9(2.2)$ & \\
\hline \multirow{4}{*}{ Age } & $15-29$ & $95(22.9)$ & $9(2.2)$ & 0.288 \\
\hline & $30-44$ & $98(23.6)$ & $6(1.4)$ & \\
\hline & $45-59$ & $98(23.6)$ & $6(1.4)$ & \\
\hline & $>60$ & $100(24.1)$ & $3(0.7)$ & \\
\hline \multirow[t]{4}{*}{ Marital Status } & Single & $100(24.1)$ & $4(1.0)$ & 0.183 \\
\hline & Married & $249(60.0)$ & $20(4.8)$ & \\
\hline & Divorced & $10(2.4)$ & $0(0.0)$ & \\
\hline & Widowed & $32(7.7)$ & $0(0.0)$ & \\
\hline \multirow[t]{7}{*}{ Educational attainment } & Less than primary school & $8(1.9)$ & $2(0.5)$ & 0.550 \\
\hline & Primary school & $181(43.6)$ & $11(2.7)$ & \\
\hline & Junior High School & $83(20.0)$ & $6(1.4)$ & \\
\hline & High school diploma & $76(18.3)$ & $4(1.0)$ & \\
\hline & Diploma/Vocational certificate & $17(4.1)$ & $0(0.0)$ & \\
\hline & Bachelor's degree & $23(5.5)$ & $1(0.2)$ & \\
\hline & Post-graduate degree & $3(0.7)$ & $0(0.0)$ & \\
\hline \multirow[t]{8}{*}{ Occupation } & Farmer & $121(29.2)$ & $10(2.4)$ & 0.711 \\
\hline & Contractors & $82(19.7)$ & $6(1.4)$ & \\
\hline & Trade & $68(16.4)$ & $3(0.7)$ & \\
\hline & Employees & $15(3.6)$ & $0(0.0)$ & \\
\hline & Government officials & $17(4.1)$ & $2(0.5)$ & \\
\hline & Housewife & $47(11.3)$ & $3(0.7)$ & \\
\hline & Student & $29(7.0)$ & $0(0.0)$ & \\
\hline & Others & $12(2.9)$ & $0(0.0)$ & \\
\hline \multirow[t]{4}{*}{ The income per month (Thai baht) } & $<1,000$ & $68(16.4)$ & $2(0.5)$ & 0.643 \\
\hline & $1,001-3,000$ & $61(14.7)$ & $3(0.7)$ & \\
\hline & $3,001-5,000$ & $64(15.4)$ & $4(1.0)$ & \\
\hline & $>5,000$ & $198(47.7)$ & $15(3.6)$ & \\
\hline
\end{tabular}

\section{Discussion}

Although the social age has evolved, some people's lifestyles are still unchanged, and precautionary eating habits of people in Ubon Ratchathani Province, Northeast Thailand, have remained consistent. Many people still consume raw fish or even raw fermented fish, which puts them at risk for opisthorchiasis infection. These food traditions may have continued due to the way of life, society, and beliefs about food consumption. People who consume raw freshwater fish are still in danger of developing opisthorchiasis. In this study, $94.2 \%$ of
Participants reported good preventive behavior for opisthorchiasis and good knowledge (80.5\%) about opisthorchiasis, respectively. Similar to our findings, Songserm et al. [27] reported that the majority of the local participants in Ubon Ratchathani, the alluvial plains of Northeast Thailand, are eating uncooked fermented fish Koi Pla (raw fish salad) or Pla Jom (pickled fish). Chuangchaiya et al. [18], opisthorchiasis was found to have a statistically significant relationship with "the habit of consuming unsafely prepared fish, the similar habit 
of family members, history of opisthorchiasis, history of ingestion of praziquantel and unsafe disposal of food waste". This study considered that all participants are from rural Thailand. Therefore, the difference in the consumption behavior of the raw food (fish) based on residency was not discussed. However, the study results showed no significant relationship between the participants' characteristics and the behavior associated with the prevention of opisthorchiasis ( $>0.05)$. Suwannahitatorn et al. [28] found that despite O. viverrini's not endemic in central Thailand, the prevalence was quite high in rural areas. Indeed, changing the consumption of the popular local delicacy raw fish among the rural population is a difficult task because of the cultural-related lifestyle passed down from their forefathers to their children [29,30]. Songserm et al. [31] examined the variables that may influence community participation in cholangiocarcinoma prevention. The authors found that the effect of official health guidelines was positive in about sixty percent of the rural population in wetland areas of Ubon Ratchathani, Thailand, who regularly participated in cholangiocarcinoma prevention activities. Unlike our findings, Songserm et al. [29] found that variables such as age and healthy behavior to prevent cholangiocarcinoma were significantly associated with community participation $(p<0.001)$. Sriamporn et al. [15] found "a positive relationship between the prevalence of opisthorchiasis infection and incidence of cholangiocarcinoma at the population level". Previous studies conducted by Saengsawang et al. [16] and Kaewpitoon et al. [17], Chuangchaiya et al. [18], and Chudthaisong et al. [19] found that females were slightly less likely to be infected than their counterparts, and infection was positively related to age in both genders. Moreover, Kaewpitoon et al. [17] reported a positive association between the prevalence of opisthorchiasis and respondents' level of education and occupation. Moreover, Chuangchaiya et al. [18] found that the prevalence of opisthorchiasis is significantly related to the history of eating raw fish. Although education level was not significantly related to knowledge in our study, Kitphati et al. [32] reported that education significantly helps in increasing the participants' knowledge and awareness towards the preventive behavior of Opisthorchiasis among about seventy percent of respondents. Moreover, the authors found that rural residents in the lower north Thailand ate Koi Pla, heavily fermented fish (Pla Ra), and short pickled fish as the main element in food in Phitsanulok province. In this study, there was no association between Opisthorchiasis and the income level; however, Kaewpitoon et al. [33 ] found that most of the surveyed people were Males, old age group, at primary school, working in the agricultural field and, and earing an income less than 4,000 baht per month.

\section{Limitation of study}

First, First, we did not have information about the nonresponders and the possibility that they differed in some criteria from the respondents, so we believe that response bias is possible. Second A cross-sectional study cannot establish a causal relationship between variables.

\section{Conclusion}

The study shows the majority of our participants reported a good level of prevention behavior for opisthorchiasis (94.2\%) and a good level of knowledge to prevent opisthorchiasis $(80.5 \%)$. In our study, participant personal characteristics, such as sex, age, the status of marriage, amount of education, occupation, and income, were not related to the level of opisthorchiasis prevention behavior. We recommend that public health officers promote accurate knowledge and prevention behavior about opisthorchiasis regularly. Public health officers should also increase public awareness about how eating raw seafood increases the risk of opisthorchiasis, leading to cholangiocarcinoma.

\section{Abbreviation}

LAO PDR: Lao People's Democratic Republic; DNA: Deoxyribonucleic acid; O. viverrini: Opisthorchis viverrine

\section{Declaration}

\section{Acknowledgment}

We would like to thank people at the That sub-district, Warin Chamrap district of the Ubon Ratchathani province, Thailand, for the cooperation in this research and thank you for scientific editing by Dr. Elizabeth Kelly Hom Thepaksorn, Director of English Editing Service Center, Sirindhorn College of Public Health, Trang, Thailand, and Dr. Phayong Thepaksorn, Trang, Research Center for Occupational Health Sirindhorn College of Public Health, Trang, Thailand.

\section{Funding}

The authors received no financial support for their research, authorship, and/or publication of this article.

Availability of data and materials

Data will be available by emailing kaewjai@scphub.ac.th.

\section{Authors' contributions}

Kaewjai Maleelai (KM) is the principal investigator of this manuscript who designed the study and coordinated all aspects of the research, including analysis, interpretation of data, drafting the work, writing the manuscript, reviewing, and approving the manuscript. Sasimaphon Chaikaew, Aungsumalin Nakham, and Jinda Khumkaew designed the study, data collection, analysis, and interpretation of data. All authors have read and approved the final manuscript.

\section{Ethics approval and consent to participate}

We conducted the research following the Declaration of Helsinki. The ethical protocol was approved by the Ethics Committee of Sirindhorn College of Public Health, Ubon Ratchathani, Thailand (Ref: SCPHUBI 012/2561, 2018). Moreover, written informed consent was obtained from each participant after explaining the study objectives and the guarantee of secrecy.

Consent for publication

Not applicable

Competing interest

The authors declare that they have no competing interests.

Open Access 
This article is distributed under the terms of the Creative Commons Attribution 4.0 International License (http://creativecommons.org/licenses/by/4.0/), which permits unrestricted use, distribution, and reproduction in any medium, provided you give appropriate credit to the original author(s) and the source, provide a link to the Creative Commons license, and indicate if changes were made. The Creative Commons Public Domain Dedication waiver (http://creativecommons.org/publicdomain/zero/1.0/) applies to the data made available in this article unless otherwise stated.

\section{Author Details}

${ }^{1}$ Sirindhorn College of Public Health, Ubon Ratchathani, Faculty of Public Health and Allied Health Sciences, Praboromarajchanok Institute, Thailand. ${ }^{2}$ Ban Muang Phrai Health Promoting Hospital, Seraphim district, Roi Et province, Thailand. ${ }^{3}$ Talsum Hospital, Talcum district, Ubon Ratchathani province, Thailand.

\section{Article Info}

Received: 16 August 2021

Accepted: 14 November 2021

Published: 23 November 2021

\section{References}

1. Centers for Disease Control and Prevention. Opisthorchiasis. Available from: : ://www.cdc.gov/dpdx/opisthorchiasis/index.html [Accessed on 7 November 2021].

2. Pengput A, Schwartz DG. Risk factors for Opisthorchis viverrini infection: A systematic review. J Infect Public Health. $2020 \quad$ Sep;13(9):1265-1273. doi: 10.1016/j.jiph.2020.05.028.

3. Boondit J, Suwannahitatorn P, Siripattanapipong S, Leelayoova S, Mungthin M, Tan-Ariya P, Piyaraj P, Naaglor T, Ruang-Areerate T. An epidemiological survey of Opisthorchis viverrini infection in a lightly infected community, eastern Thailand. Am J Trop Med Hyg. 2020 Apr;102(4):838-843. https://doi.org/10.4269/ajtmh.190864.

4. Kaewpitoon N, Kaewpitoon SJ, Pengsaa P, Sripa B. Opisthorchis viverrini: the carcinogenic human liver fluke. World J Gastroenterol. 2008;14(5):666-74. doi: 10.3748/wjg.14.666.

5. Sripa B, Tangkawattana S, Laha T, Kaewkes S, Mallory FF, Smith JF, Wilcox BA. Toward integrated opisthorchiasis control in northeast Thailand: The Lawa project. Acta Trop. 2015 Jan;141(Pt B):361-7. doi: 10.1016/j.actatropica.2014.07.017

6. Saichua P, Sithithaworn P, Jariwala AR, Diemert DJ, Sithithaworn J, Sripa B, et al. Microproteinuria during Opisthorchis viverrini infection: a biomarker for advanced renal and hepatobiliary pathologies from chronic opisthorchiasis. PLoS Negl Trop Dis. 2013;7(5): e2228. https://doi: 10.1371/journal.pntd.0002228.

7. Center of Disease Control and Prevention. 2019. Liver Flukes. Available from: https://www.cdc.gov/parasites/liver_flukes/ [Accessed on 5 March 2021].
8. Sripa B, Pairojkul C. Cholangiocarcinoma: lessons from Thailand. Curr Opin Gastroenterol. 2008;24(3):349-56. https://doi: 10.1097/MOG.0b013e3282fbf9b3.

9. Dao TT, Bui TV, Abatih EN, Gabriël S, Nguyen TT, Huynh QH, et al. Opisthorchis viverrini infections and associated risk factors in a lowland area of Binh Dinh province, central Vietnam. Acta Trop. 2016 May; 157:1517. doi: 10.1016/j.actatropica.2016.01.029.

10. Hughes T, O'Connor T, Techasen A, Namwat N, Loilome $\mathrm{W}$, Andrews RH, et al. Opisthorchiasis and cholangiocarcinoma in Southeast Asia: an unresolved problem. Int J Gen Med. 2017; 10:227-237. https://doi: 10.2147/IJGM.S133292.

11. Wattanayingcharoenchaia S, Nithikathkulb C, Wongsarojc $\mathrm{T}$, Royald L, Reungsange P. Geographic information system of Opisthorchis viverrini in northeast Thailand. Asian Biomedicine; 2011;5(5):687-691. https://doi.org/10.5372/1905-7415.0505.090

12. Andrews RH, Sithithaworn P, Petney TN. Opisthorchis viverrini: an underestimated parasite in world health. Trends Parasitol. 2008;24(11):497-501. doi: 10.1016/j.pt.2008.08.011.

13. Sripa B, Bethony JM, Sithithaworn P, Kaewkes S, Mairiang E, Loukas A, Mulvenna J, Laha T, Hotez PJ, Brindley PJ. Opisthorchiasis and Opisthorchis-associated cholangiocarcinoma in Thailand and Laos. Acta Trop. 2011 Sep;120 Suppl 1(Suppl 1): S158-68. doi: 10.1016/j.actatropica.2010.07.006.

14. Xia J, Jiang S-c, Peng H-J. Association between liver fluke infection and hepatobiliary pathological changes: A systematic review and meta-analysis. PLoS ONE. 2015;10(7): $\quad$ e0132673. https://doi.org/10.1371/journal.pone.0132673

15. Sriamporn S, Pisani P, Pipitgool V, Suwanrungruang K, Kamsa-ard S, Parkin DM. Prevalence of Opisthorchis viverrini infection and incidence of cholangiocarcinoma in Khon Kaen, Northeast Thailand. Trop Med Int Health. 2004 May;9(5):588-94.https://doi.org/10.1111/j.13653156.2004.01234.x

16. Saengsawang $P$, Promthet $S$, Bradshaw P. Prevalence of $\mathrm{OV}$ infection in Yasothon province, northeast Thailand. Asian Pac J Cancer Prev. 2012;13(7):3399-402. https://doi:10.7314/apjcp.2012.13.7.3399.

17. Kaewpitoon SJ, Rujirakul R, Kaewpitoon N. Prevalence of Opisthorchis viverrini infection in Nakhon Ratchasima province, northeast Thailand. Asian Pac J Cancer Prev. 2012;13(10):5245-9. https://doi:10.7314/apjcp.2012.13.10.5245.

18. Chuangchaiya S, Navanesan S, Jaichuang S, Rahim MAFA, Idris ZM. Current prevalence of Opisthorchis viverrini infection and associated risk factors in Nakhon Phanom province, northeastern Thailand. Trop Biomed. 2020 Dec;37(4):986-999. doi: 10.47665/tb.37.4.986.

19. Chudthaisong N, Promthet S, Bradshaw P. Risk factors for Opisthorchis viverrini infection in Nong Khai province, Thailand. Asian Pacific Journal of Cancer Prevention. 2015;16. 4593-4596. doi: 10.7314/apjcp.2015.16.11.4593

20. Prakobwong S, Suwannatrai A, Sancomerang A, Chaipibool S, Siriwechtumrong N. A largescale study of the epidemiology and risk factors for the carcinogenic liver 
fluke Opisthorchis viverrini in Udon Thani province, Thailand. Asian Pac J Cancer Prev. 2017;18 (10), 28532860 doi:10.22034/APJCP.2017.18.10.2853

21. Songserm N, Woradet S, Bureelerd O, Charoenbut P. Evaluation of Cholangiocarcinoma risk and its related factors in wetland Geographical communities of Ubon Ratchathani, Thailand. Asian Pac J Cancer Prev. 2016;17(4):1811-5. https://doi.org/10.7314/APJCP.2016.17.4.1811

22. Kaewpitoon N, Kaewpitoon SJ, Pengsaa P. Ophistorchiasis in Thailand: Review and current status. World J Gastroenterol 2008; 14(15): 2297-2302 doi:10.3748/wjg.14.2297.

23. Srithai C, Chuangchaiya S, Jaichuang S, Idris ZM. Prevalence of Opisthorchis viverrini and its associated risk factors in the Phon Sawan District of Nakhon Phanom province, Thailand. Iran J Parasitol. 2021 JulSep;16(3):474-482. doi:10.18502/ijpa.v16i3.7101.

24. Thaewnongiew K, Singthong S, Kutchamart S, Tangsawad S, Promthet S, Sailugkum S, Wongba N. Prevalence and risk factors for Opisthorchis viverrini infections in upper northeast Thailand. Asian Pac J Cancer Prev. 2014;15(16):6609-12. https://doi.org/10.7314/APJCP.2014.15.16.6609

25. Khuntikeo N, Sithithaworn P, Loilom W, Namwat N, Yongvanit P, Thinkhamrop B, Kiatsopit N, Andrews RH, Petney TN. Changing patterns of prevalence in Opisthorchis viverrini sensu lato infection in children and adolescents in northeast Thailand. Acta Trop. 2016 Dec; 164:469-472. doi: 10.1016/j.actatropica.2016.10.017.

26. Prompolmuang $\mathrm{K}$ and Kessomboon P. Risk factors to Opisthorchiasis among people in Sriboonruang District, Nongbualamphu Province. Graduate research conference, Khon Khaen University 2014. MMP91. p.1882-1888. Available from: http://gsbooks.gs.kku.ac.th/57/grc15/files/mmp91.pdf

27. Songserm N, Woradet S, Sripa B, Ali A. Sustainable prevention of cholangiocarcinoma through community participation in a high-incidence area in Thailand. Asian Pac J Cancer Prev. 2020;21(3),777-782. doi:10.31557/APJCP.2020.21.3.777

28. Suwannahitatorn P, Webster J, Riley S, Mungthin M, Donnelly CA. Uncooked fish consumption among those at risk of Opisthorchis viverrini infection in central Thailand. PLoS ONE. 2019;14(1): e0211540. https://doi.org/10.1371/journal.pone.0211540

29. Chuangchaiya S, Laoprom N, Idris ZM. Prevalence and associated risk factors of Opisthorchis viverrini infections in rural communities along the Nam Kam river of northeastern Thailand. Trop Biomed. 2019;36(1):81-93.

30. Chaiputcha K, Promthet S, Bradshaw P. Prevalence and Risk Factors for Infection by Opisthorchis viverrini in an urban area of Mahasarakham province, northeast Thailand. Asian Pac J Cancer Prev. 2015;16(10):4173-6. https://doi.org/10.7314/APJCP.2015.16.10.4173

31. Songserm N, Bureelerd O, Thongprung S, Woradet S, Promthet S. Community participation in cholangiocarcinoma prevention in Ubon Ratchathani, Thailand: relations with age and health behavior. Asian Pac J Cancer Prev. 2015;16(16):7375-7379. http://dx.doi.org/10.7314/APJCP.2015.16.16.7375

32. Kitphati R, Seangpraw K, Wattanawong O, Choowanthanapakorn M, Wongsaroj T. Community participation for behavior development to prevent opisthorchiasis among people in a rural area of lower north Thailand. Journal of Health Research, 2017;231(5):347354. https://he01.tcithaijo.org/index.php/jhealthres/article/view/99559

33. Kaewpitoon SJ, Rujirakul R, Wakkuwattapong R, Matrakool L, Tongtawee T, Panpimanmas S, Pengsaa P, Jomkoa D, Joosiri A, Kaewpitoon N. Opisthorchis viverrini Infection Among People in the Border Areas of Three Provinces, Northeast of Thailand. Asian Pac J Cancer Prev. 2016;17(6):2973-7. 